\title{
Global end-diastolic volume increases to maintain fluid responsiveness in sepsis-induced systolic dysfunction
}

\author{
Ronald J Trof ${ }^{1,2}$, Ibrahim Danad ${ }^{1}$ and AB Johan Groeneveld ${ }^{1,3^{*}}$
}

\begin{abstract}
Background: Sepsis-induced cardiac dysfunction may limit fluid responsiveness and the mechanism thereof remains unclear. Since cardiac function may affect the relative value of cardiac filling pressures, such as the recommended central venous pressure (CVP), versus filling volumes in guiding fluid loading, we studied these parameters as determinants of fluid responsiveness, according to cardiac function.
\end{abstract}

Methods: A delta CVP-guided, 90 min colloid fluid loading protocol was performed in 16 mechanically ventilated patients with sepsis-induced hypotension and three 30 min consecutive fluid loading steps of about $450 \mathrm{~mL}$ per patient were evaluated. Global end-diastolic volume index (GEDVI), cardiac index (Cl) and global ejection fraction (GEF) were assessed from transpulmonary dilution. Baseline and changes in CVP and GEDVI were compared among responding $(\mathrm{Cl}$ increase $\geq 10 \%$ and $\geq 15 \%)$ and non-responding fluid loading steps, in patient with low $(<20 \%, \mathrm{n}=9)$ and near-normal ( $\geq 20 \%) \mathrm{GEF}(n=7)$ at baseline.

Results: A low GEF was in line with other indices of impaired cardiac (left ventricular) function, prior to and after fluid loading. Of 48 fluid loading steps, 9 (of 27) were responding when GEF $<20 \%$ and 6 (of 21) when GEF $\geq 20$. Prior to fluid loading, CVP did not differ between responding and non-responding steps and levels attained were 23 higher in the latter, regardless of GEF ( $P=0.004$ ). Prior to fluid loading, GEDVI (and $\mathrm{Cl}$ ) was higher in responding $\left(1007 \pm 306 \mathrm{~mL} / \mathrm{m}^{2}\right)$ than non-responding steps $\left(870 \pm 236 \mathrm{~mL} / \mathrm{m}^{2}\right)$ when GEF was low $(P=0.002)$, but did not differ when GEF was near-normal. Increases in GEDVI were associated with increases in $\mathrm{Cl}$ and fluid responsiveness, regardless of GEF $(P<0.001)$.

Conclusions: As estimated from transpulmonary dilution, about half of patients with sepsis-induced hypotension have systolic cardiac dysfunction. During dysfunction, cardiac dilation with a relatively high baseline GEDVI maintains fluid responsiveness by further dilatation (increase in GEDVI rather than of CVP) as in patients without dysfunction. Absence of fluid responsiveness during systolic cardiac dysfunction may be caused by diastolic dysfunction and/or right ventricular dysfunction.

Keywords: Septic Shock, Fluid Loading, Myocardial Depression, Right Ventricular Afterload, Diastolic Compliance, Filling Volumes, Ejection Fraction

\footnotetext{
* Correspondence: a.b.j.groeneveld@erasmusmc.nl

'Department of Intensive Care, Vrije Universiteit Medical Centre, Amsterdam,

The Netherlands

${ }^{3}$ Department of Intensive Care, Erasmus Medical Centre, Rotterdam, The

Netherlands

Full list of author information is available at the end of the article
} 


\section{Background}

Patients with severe sepsis or septic shock commonly develop cardiac dysfunction, even in the absence of cardiac ischemia [1-3]. These abnormalities may include depression of left and/or right ventricular systolic function and/or diastolic dysfunction and may be accompanied by ventricular dilatation, as estimated from echocardiography or radionuclide cineangiography $[4,5]$. This cardiac dysfunction is usually reversible and returns to normal in 7 to 10 days in survivors [6-8]. Systolic dysfunction-induced ventricular dilatation is suggested to be an adaptive mechanism to maintain a high cardiac output which is associated with survival $[4,9]$, while other investigators denied such a dilatory response arguing in favor of impaired relaxation and diastolic (often upon systolic) dysfunction contributing to non-survival [8,10-16].

Fluid loading is often the initial treatment of sepsisinduced hypotension and the response may be diminished in sepsis-induced cardiac depression associated with severe disease and non-survival $(5,13,14)$. On the other hand, fluid overloading when the heart is non-responsive and the central venous pressure (CVP) is inadvertently elevated is potentially harmful and also associated with mortality, emphasizing the value of appropriate haemodynamic monitoring [17]. By optimizing preload and assessing fluid responsiveness, deleterious hypoperfusion and fluid overloading may be prevented. Traditionally, filling pressures, like CVP, have been used to guide fluid loading in sepsis-induced hypotension [17-20], even though its predictive value for fluid responsiveness during mechanical ventilation and altered cardiac function is doubtful [21-23]. Alternatively, the transpulmonary dilution technique estimates the global end-diastolic volume index (GEDV), and pulmonary blood volume index (PBVI) as a superior and global measures of cardiac preload [11,23,24]. The GEDVI represents the volumes of the right and left heart at the end of diastole and often reflects left ventricular end-diastolic volume estimated by echocardiography provided that right ventricular dilatation is absent [25].

A relatively low GEDVI may predict fluid responsiveness (and a relatively high GEDVI absence thereof), but the role of systolic and/or diastolic dysfunction with respect to interpretation of absolute values remains unclear, even though changes in stroke volume or cardiac output correlate to changes in GEDVI $[21,23,26]$. Indeed, the relative value of GEDVI and filling pressures in determining fluid responsiveness depends on systolic cardiac function, at least in non-septic patients [27]. Conversely, echocardiographic end-diastolic left ventricular dimensions poorly predicted fluid responsiveness but changes were superior to filling pressures in monitoring changes in cardiac output upon fluid loading in some studies on sepsis $[9,23]$. In contrast, fluid responsiveness was found to be associated with biventricular dilatation by nuclear angiography and non-responsiveness appeared attributable to right ventricular systolic dysfunction following mild pulmonary hypertension in other studies on sepsis $[6,9]$.

In view of the above controversies on mechanisms and predictive values, we evaluated and compared filling volumes to pressures in determining the cardiac response to fluid loading according to systolic cardiac function in sepsis-induced hypotension, in the hypothesis that, even in dysfunctioning hearts, cardiac dilatation is required to increase cardiac output upon fluid loading.

\section{Methods}

This was a sub-study of a prospective, non-randomized, single-center clinical trial, investigating the cardiorespiratory effects of various resuscitation fluids in presumed hypovolemia during sepsis and non-sepsis, in mechanically ventilated patients in the intensive care unit (ICU) [24,28]. We analyzed, retrospectively, 16 patients with sepsis monitored by both CVP and the transpulmonary dilution technique. These patients were divided in two groups according to a low GEF $(<20 \%)$ and near-normal GEF ( $\geq 20 \%$ ). The cutoff of $20 \%$ approximately reflects a cutoff of $40 \%$ ejection fraction of the left ventricle as measured by echocardiography, provided that there is no right ventricular dysfunction [29-31]. The original study was approved by the Ethics Committee of the Vrije Universiteit Medical Center and written informed consent was obtained. We analyzed the effect of colloid fluid loading in patients with sepsis-induced hypotension. Colloid fluid loading was given with modified fluid gelatin $4 \%$, hydroxyethyl starch (HES) 6\% or albumin 5\%, all of which have similar oncotic properties and haemodynamic responses $[24,28,32]$. We only analyzed patients who completed fluid loading and measurements up to $t=90 \mathrm{~min}$. Inclusion criteria, at enrollment and start of the protocol, were clinical criteria for presumed hypovolemia commonly triggering fluid infusion, such as a relatively arbitrarily chosen systolic blood pressure $<110 \mathrm{mmHg}$ and a low CVP, roughly taking transmission of positive end-expiratory pressure (PEEP) into account (Table 1). Exclusion criteria were age $>75$ year, preterminal illness with a life expectancy of less than 24 hours, or known anaphylactic reactions to colloids. Sepsis was defined according to international guidelines [33]. The origin of sepsis was defined by clinical signs and symptoms, imaging techniques and positive local and/or blood cultures [33]. All patients were on controlled mechanical ventilation and positive end-expiratory pressure (PEEP).

\section{Study protocol}

The protocol was started in the ICU when patients met the inclusion criteria. Demographic characteristics were recorded, including the acute physiology and chronic health evaluation (APACHE-II). After baseline measurements 
Table 1 Fluid challenge protocol

\begin{tabular}{|c|c|c|}
\hline \multirow[t]{6}{*}{ CVP at start: } & $\leq 8$ if $\mathrm{PEEP} \leq 15$ & $200 \mathrm{ml} / 10 \mathrm{~min}$ \\
\hline & $\leq 12$ if PEEP $>15$ & $200 \mathrm{ml} / 10 \mathrm{~min}$ \\
\hline & $\leq 10$ if PEEP $\leq 15$ & $100 \mathrm{ml} / 10 \mathrm{~min}$ \\
\hline & $\leq 14$ if $P E E P>15$ & $100 \mathrm{ml} / 10 \mathrm{~min}$ \\
\hline & $\leq 12$ if $P$ EEP $\leq 15$ & $50 \mathrm{ml} / 10 \mathrm{~min}$ \\
\hline & $\leq 16$ if $\mathrm{PEEP}>15$ & $50 \mathrm{ml} / 10 \mathrm{~min}$ \\
\hline CVP during infusion: & increase $>5$ & stop \\
\hline \multirow[t]{3}{*}{ CVP after 10 min: } & increase $\leq 2$ & continue \\
\hline & $2<$ increase $\leq 5$ & wait $10 \mathrm{~min}$ \\
\hline & increase $>5$ & stop \\
\hline \multirow[t]{2}{*}{ CVP after 10 min waiting: } & increase $>2$ & stop \\
\hline & increase $\leq 2$ & repeat \\
\hline
\end{tabular}

CVP Central venous pressure $(\mathrm{mmHg})$, PEEP Positive end-expiratory pressure $\left(\mathrm{cm} \mathrm{H}_{2} \mathrm{O}\right)$.

were taken, fluids were given over 90 min on the basis of the response within predefined limits of increases in CVP, according to a previously described protocol (Table 1 ) $[24,28,32]$. Up to $200 \mathrm{~mL}$ of fluid were given every $10 \mathrm{~min}$, provided that the increase in CVP upon fluid loading did not exceed critical values, and this policy has been proven safe in previous studies (i.e. not evoking pulmonary edema) $[24,28]$. The maximum amount of fluid infused was $1800 \mathrm{~mL}$. Fluid responsiveness was defined as an increase of $\mathrm{CI} \geq 10$ and $15 \%$, in accordance with the literature $[22,33]$, between $t=0-30, t=30-60$ and $t=60-90$ min upon fluid loading. Concomitant vasoactive and sedative drug treatment and ventilatory settings remained unchanged during fluid loading.

\section{Measurements}

Heart rate (HR) and mean arterial pressure (MAP) were recorded at $\mathrm{t}=0$ and $90 \mathrm{~min}$. MAP and CVP were measured in the supine position after calibration, zeroing to atmospheric pressure at the midchest level at end-expiration (Tramscope ${ }^{\mathrm{R}}$, Marquette GE, Milwaukee, Wisconsin). Cardiac output, GEDVI, PBVI and CVP were measured every $30 \mathrm{~min}$, from $\mathrm{t}=0$ to $90 \mathrm{~min}$. Relevant measurements were indexed to body surface area (BSA), giving stroke volume index $\left(\mathrm{SVI}, \mathrm{mL} / \mathrm{m}^{2}\right)$, cardiac index $\left(\mathrm{CI}, \mathrm{L} / \mathrm{min} / \mathrm{m}^{2}\right)$, GEDVI (n $680-800 \mathrm{~mL} / \mathrm{m}^{2}$ ) and PBVI (n $150-250 \mathrm{~mL} / \mathrm{m}^{2}$ ), respectively. For these measurements, the transpulmonary thermal-dye indicator dilution technique was used (11). These measurements involve averages obtained from 2-3 central venous injections of $15 \mathrm{~mL}$ of ice-cold indocyanine green in 5\% glucose solution and concomitant registration of the dilution curves in the femoral artery, by a $3 \mathrm{~F}$ catheter equipped with a thermistor (PV 2024, Pulsion Medical Systems, Munich, Germany). This catheter was inserted via a $4 \mathrm{~F}$ introducing sheath (Arrow, Reading, USA) and connected to a bedside computer (COLD Z-021, Pulsion
Medical Systems, Munich, Germany. The COLD Z-021 is the precursor to the current transpulmonary thermodilution pulse contour cardiac output $\left(\mathrm{PiCCO}^{\mathrm{mm}}\right)$ technique and yields the same cardiac parameters. Reproducibility of measurements is typically within 10\% (11). GEDVI represents the volumes of the right and left heart at end-diastole and reflects left ventricular dimensions obtained by echocardiography in the absence of overt right ventricular distention (25). The ratio between stroke volume index (cardiac index/HR) and GEDVI/4 is defined as the global ejection fraction (GEF, normal values $25-35 \%$ ), and is an indicator of left ventricular systolic function, provided that there is no right ventricular dysfunction (29-31). Left ventricular stroke work index (LVSWI, $\mathrm{gm} / \mathrm{m}^{2}$ ) was calculated from SVI x (MAP-CVP) x 0.0136 and cardiac function index (CFI, n 18.0-26.0 1/min) from CI/(GEDVI/4) $(30,31)$. Preload-recruitable stroke work was defined by LVSWI/GEDVI (24). CFI, LVSWI and LVSWI/GEDVI were used to assess cardiac (e.g. left ventricular) systolic function. The lung injury score was calculated from radiographic densities, oxygenation ratio $\mathrm{P}_{\mathrm{a}} \mathrm{O}_{2} / \mathrm{F}_{\mathrm{I}} \mathrm{O}_{2}, \mathrm{PEEP}$ and dynamic compliance and ranges between $0-4$. Mortality refers to death in the ICU.

\section{Statistical analysis}

For categorical data, Fisher exact tests were used. Since continuous data were normally distributed (KolmogorovSmirnov test, $P>0.05$ ), they were summarized by mean \pm standard deviation (SD) and parametric tests were done. Paired and unpaired t-tests were used to compare data in time and between GEF groups, respectively generalized estimating equations (GEE) were used to evaluate differences in baseline and changes in variables between summated responding and non-responding fluid loading steps in each GEF group, to evaluate their determining values, respectively, taking repeated measurements in the same patients and type and volume of fluid administered (as covariates) into account. Exact two-sided P values $>0.001$ are given and considered statistically significant when $<0.05$. All analyses were conducted using SPSS version 15.0 (SPSS Chicago, Ill, USA).

\section{Results}

Table 2 summarizes the characteristics of patients. The haemodynamic variables differ according to GEF and changes upon fluid loading. There was no difference in the amount and type of fluids infused between the GEF groups. GEF did not change during fluid loading. In the low GEF group, other function indices also pointed to systolic cardiac dysfunction, prior to and after fluid loading, even though the $\mathrm{CI}$ attained with fluid loading did not differ among the groups. The number of fluid loading responses did not differ according to GEF, but the increase in CI decreased with increasing fluid loading steps only when 
Table 2 Patient characteristics

\begin{tabular}{|c|c|c|c|}
\hline & GEF $<20 \%$ & $\mathrm{GEF} \geq \mathbf{2 0} \%$ & \\
\hline & $(n=9)$ & $(n=7)$ & $P$ value \\
\hline Age & $62 \pm 9$ & $57 \pm 9$ & 0.32 \\
\hline Male/female & $7 / 2$ & $4 / 3$ & 0.60 \\
\hline APACHE $\|$ & $16 \pm 4$ & $12 \pm 5$ & 0.08 \\
\hline Cardiac premorbidity & 4 & 1 & 0.31 \\
\hline Sepsis origin & & & 0.38 \\
\hline pulmonary & 4 & 3 & \\
\hline abdominal & 2 & 0 & \\
\hline CNS & 0 & 1 & \\
\hline urogenital & 1 & 0 & \\
\hline unknown & 2 & 3 & \\
\hline Bloodstream infection & & & 0.41 \\
\hline Gram- & 2 & & \\
\hline Gram+ & 2 & 2 & \\
\hline Fungi & & 1 & \\
\hline PEEP, $\mathrm{cm} \mathrm{H}_{2} \mathrm{O}$ & $14 \pm 6$ & $12 \pm 3$ & 0.17 \\
\hline Tidal volume, mL/kg & $8.0 \pm 0.8$ & $9.0 \pm 1.6$ & 0.08 \\
\hline $\mathrm{P}_{\mathrm{a}} \mathrm{O}_{2} / \mathrm{F}_{1} \mathrm{O}_{2}$ & $209 \pm 54$ & $193 \pm 62$ & 0.60 \\
\hline Lung injury score & $2.2 \pm 0.8$ & $2.5 \pm 0.8$ & 0.60 \\
\hline ICU mortality & 4 & 2 & 0.37 \\
\hline \multicolumn{4}{|l|}{ Haemodynamics } \\
\hline \multicolumn{4}{|l|}{$\mathrm{HR}$, /min } \\
\hline$t=0$ & $106 \pm 18$ & $90 \pm 25$ & 0.15 \\
\hline$t=90$ & $103 \pm 16$ & $95 \pm 22$ & 0.42 \\
\hline \multicolumn{4}{|l|}{ MAP, mm Hg } \\
\hline$t=0$ & $73 \pm 12$ & $74 \pm 9$ & 0.84 \\
\hline$t=90$ & $88 \pm 19^{1}$ & $89 \pm 13^{1}$ & 0.87 \\
\hline \multicolumn{4}{|l|}{ CVP, mm Hg } \\
\hline$t=0$ & $9 \pm 5$ & $8 \pm 3$ & 0.61 \\
\hline$t=90$ & $12 \pm 5^{2}$ & $12 \pm 3^{2}$ & 0.83 \\
\hline \multicolumn{4}{|l|}{$\mathrm{Cl}, \mathrm{L} / \mathrm{min}$} \\
\hline$t=0$ & $3.3 \pm 0.6$ & $4.3 \pm 1.5$ & 0.06 \\
\hline$t=90$ & $3.9 \pm 1.0^{2}$ & $5.0 \pm 1.4^{3}$ & 0.09 \\
\hline \multicolumn{4}{|l|}{$\mathrm{SVI}, \mathrm{mL} / \mathrm{m}^{2}$} \\
\hline$t=0$ & $31 \pm 6$ & $49 \pm 12$ & 0.002 \\
\hline$t=90$ & $38 \pm 9^{1}$ & $53 \pm 11$ & 0.01 \\
\hline \multicolumn{4}{|l|}{ LVSWI, gm/m² } \\
\hline$t=0$ & $27 \pm 5$ & $43 \pm 8$ & $<0.001$ \\
\hline$t=90$ & $39 \pm 11^{3}$ & $55 \pm 11^{4}$ & 0.01 \\
\hline \multicolumn{4}{|l|}{ GEDVI, $\mathrm{mL} / \mathrm{m}^{2}$} \\
\hline$t=0$ & $891 \pm 257$ & $787 \pm 140$ & 0.35 \\
\hline$t=90$ & $963 \pm 273^{1}$ & $866 \pm 170^{1}$ & 0.43 \\
\hline \multicolumn{4}{|l|}{$\mathrm{GEF}, \%$} \\
\hline$t=0$ & $15 \pm 2$ & $25 \pm 5$ & n.a. \\
\hline
\end{tabular}

Table 2 Patient characteristics (Continued)

\begin{tabular}{llll}
\hline $\mathrm{t}=90$ & $16 \pm 4$ & $25 \pm 7$ & 0.005 \\
$\mathrm{CFI}, 1 / \mathrm{min}$ & & & \\
$\mathrm{t}=0$ & $15.2 \pm 2.9$ & $22.1 \pm 6.2$ & 0.01 \\
$\mathrm{t}=90$ & $16.8 \pm 4.0$ & $23.4 \pm 5.5$ & 0.01 \\
$\mathrm{LVSWI} / \mathrm{GEDVI}, \mathrm{gm} / \mathrm{mL}$ & & & \\
$\mathrm{t}=0$ & $0.13 \pm 0.04$ & $0.22 \pm 0.04$ & $<0.001$ \\
$\mathrm{t}=90$ & $0.17 \pm 0.07^{4,3}$ & $0.26 \pm 0.07$ & 0.02 \\
Norepinephrine and/or dopamine & 8 & 6 & 0.70 \\
Norepinephrine, $\mu \mathrm{g} / \mathrm{kg} / \mathrm{min}$ & $0.09 \pm 0.11$ & $0.06 \pm 0.12$ & 0.25 \\
Dopamine, $\mu \mathrm{g} / \mathrm{kg} / \mathrm{min}$ & $5.6 \pm 3.4$ & $4.9 \pm 4.3$ & 0.92 \\
Fluid, $\mathrm{mL}$ & $1456 \pm 296$ & $1271 \pm 269$ & 0.22 \\
Gelatin/HES/albumin & $3 / 2 / 4$ & $2 / 3 / 2$ & 0.66
\end{tabular}

Mean \pm SD or number of patients, where appropriate.

Abbreviations: GEF Global ejection fraction, APACHE // Acute physiology and chronic health evaluation, CNS Central nervous system, PEEP Positive end-expiratory pressure, ICU Intensive care unit, $\mathrm{P}_{a} \mathrm{O}_{2} / \mathrm{F}_{1} \mathrm{O}_{2}$ Arterial partial pressure of $\mathrm{O}_{2}$ over inspiratory $\mathrm{O}_{2}$ fraction, $H R$ Heart rate, MAP Mean arterial pressure, CVP Central venous pressure, $\mathrm{Cl}$ Cardiac index, SVI Stroke volume index, LVSWI Left ventricular stroke volume index, GEDVI Global end-diastolic volume, CFI Cardiac function index, HES Hydroxyethyl starch. ${ }^{1} P=0.02$,

${ }^{2} P=0.002,{ }^{3} \mathrm{P}=0.009,{ }^{3} P=0.04$ vs. $\mathrm{t}=0$, n.a. not applicable.

GEF was low $(P=0.04)$. The increases with fluids in CVP, GEDVI, MAP, LVSWI and CI did not differ among GEF groups, even though SVI, PBVI and LVSWI/GEDVI increased in the low GEF group only.

\section{Fluid loading steps in GEF groups}

Responses were independent of the type of colloid fluid, regardless of GEF and cutoff for fluid responsiveness. The CI prior to each fluid loading step was higher in responding than non-responding steps in the low GEF group, but lower in the near-normal GEF group (Table 3). The CVP did not differ between responding and nonresponding steps in both GEF groups but attained higher values after fluid loading in non-responding than in responding steps, regardless of GEF. When GEF was low, GEDVI was higher prior to responding than non-responding fluid loading steps, while GEDVI in the near-normal GEF group did not differ prior to fluid loading steps. The GEDVI and PBVI increased in responding fluid loading steps regardless of GEF. Hence, baseline CVP and GEDVI were poor predictors of fluid responsiveness in both GEF groups. When fluid responsiveness was defined as an increase in CI $\geq 15 \%$, changes in $\mathrm{CO}$ were also directly associated with changes in GEDVI, but not in PBVI. Otherwise there were only 4 out of 9 responding steps remaining when defining fluid responsiveness by 15 vs. $10 \% \mathrm{CI}$ increases, in patients with low GEF needing relatively large amounts of fluid. Baseline GEDVI was not lower in responders than non-responders. 
Table 3 Summated fluid loading steps, with responsiveness defined as $\geq 10 \%$ and $\geq 15 \%$ increase in cardiac index $(\mathrm{Cl})$, when systolic cardiac function is reduced or near-normal at $20 \%$ cutoff of global ejection fraction (GEF)

\begin{tabular}{|c|c|c|c|c|c|c|}
\hline \multirow{3}{*}{$\mathrm{Cl} \geq 10 \%$} & \multicolumn{3}{|l|}{ GEF $<20 \%(n=9)$} & \multicolumn{3}{|l|}{ GEF $\geq 20 \%(n=7)$} \\
\hline & \multirow{2}{*}{$\begin{array}{l}\mathrm{R} \\
\text { ( } \mathrm{n}=9 \text { steps in } 6 \text { patients) }\end{array}$} & \multirow{2}{*}{$\frac{N R}{(n=18 \text { steps in } 9 \text { patients })}$} & \multirow[t]{2}{*}{ P-value } & \multirow{2}{*}{$\frac{R}{(n=6 \text { stepsin } 5 \text { patients })}$} & \multirow{2}{*}{$\frac{N R}{\text { ( } n=15 \text { steps in } 7 \text { patients) }}$} & \multirow[t]{2}{*}{ P-value } \\
\hline & & & & & & \\
\hline \multicolumn{7}{|l|}{$\mathrm{Cl}, \mathrm{L} / \mathrm{min} / \mathrm{m}^{2}$} \\
\hline baseline & $3.7 \pm 0.7$ & $3.5 \pm 0.7$ & 0.04 & $3.6 \pm 1.2$ & $5.0 \pm 1.5$ & 0.008 \\
\hline after & $4.4 \pm 0.8$ & $3.4 \pm 0.6$ & & $4.3 \pm 1.4$ & $5.0 \pm 1.5$ & \\
\hline change & $0.7 \pm 0.3$ & $0 \pm 0.3$ & n.a. & $0.7 \pm 0.3$ & $0 \pm 0.2$ & n.a. \\
\hline \multicolumn{7}{|l|}{ CVP, mm Hg } \\
\hline baseline & $9 \pm 6$ & $11 \pm 5$ & 0.41 & $10 \pm 3$ & $10 \pm 3$ & 0.68 \\
\hline after & $10 \pm 6$ & $12 \pm 4$ & $10 \pm 2$ & $11 \pm 3$ & & \\
\hline change & $1 \pm 1$ & $1 \pm 2$ & $<0.001$ & $1 \pm 2$ & $2 \pm 1$ & 0.004 \\
\hline \multicolumn{7}{|l|}{ GEDVI, $\mathrm{mL} / \mathrm{m}^{2}$} \\
\hline baseline & $1007 \pm 306$ & $870 \pm 236$ & 0.002 & $801 \pm 186$ & $834 \pm 163$ & 0.83 \\
\hline after & $1102 \pm 313$ & $858 \pm 208$ & $872 \pm 199$ & $843 \pm 167$ & & \\
\hline change & $96 \pm 59$ & $-12 \pm 54$ & $<0.001$ & $70 \pm 85$ & $8 \pm 38$ & $<0.001$ \\
\hline \multicolumn{7}{|l|}{$\mathrm{PBVI}, \mathrm{mL} / \mathrm{m}^{2}$} \\
\hline baseline & $215 \pm 95$ & $203 \pm 64$ & 0.25 & $212 \pm 51$ & $225 \pm 50$ & 0.86 \\
\hline after & $250 \pm 54$ & $204 \pm 52$ & $224 \pm 40$ & $227 \pm 50$ & & \\
\hline change & $34 \pm 63$ & $1 \pm 69$ & $<0.001$ & $11 \pm 52$ & $2 \pm 53$ & $<0.001$ \\
\hline Fluid, $\mathrm{mL}$ & $522 \pm 120$ & $467 \pm 161$ & 0.07 & $450 \pm 176$ & $467 \pm 145$ & 0.75 \\
\hline \multicolumn{6}{|l|}{ albumin } & 0.24 \\
\hline \multirow[t]{2}{*}{$\mathrm{Cl} \geq 15 \%$} & $\mathrm{R}$ & NR & P-value & $\mathrm{R}$ & NR & P-value \\
\hline & ( $\mathrm{n}=4$ steps in 4 patients) & ( $\mathrm{n}=23$ steps in 9 patients) & & ( $\mathrm{n}=5$ steps in 4 patients) & ( $\mathrm{n}=16$ steps in 7 patients) & \\
\hline \multicolumn{7}{|l|}{$\mathrm{Cl}, \mathrm{L} / \mathrm{min} / \mathrm{m}^{2}$} \\
\hline baseline & $3.6 \pm 1.0$ & $3.6 \pm 0.7$ & 0.50 & $3.6 \pm 1.3$ & $4.9 \pm 1.5$ & 0.01 \\
\hline after & $4.4 \pm 1.1$ & $3.7 \pm 0$ & $4.4 \pm 1.5$ & $5.0 \pm 1.5$ & & \\
\hline change & $0.9 \pm 0.3$ & $0.1 \pm 0.3$ & na & $0.8 \pm 0.3$ & $0 \pm 0.2$ & na \\
\hline \multicolumn{7}{|l|}{$\mathrm{CVP}, \mathrm{mm} \mathrm{Hg}$} \\
\hline baseline & $10 \pm 4$ & $11 \pm 5$ & 0.59 & $9 \pm 3$ & $10 \pm 3$ & 0.61 \\
\hline after & $11 \pm 4$ & $12 \pm 5$ & $10 \pm 3$ & $11 \pm 3$ & & \\
\hline change & $1 \pm 1$ & $1 \pm 1$ & 0.76 & $1 \pm 2$ & $1 \pm 1$ & 0.05 \\
\hline \multicolumn{7}{|l|}{ GEDVI, $\mathrm{mL} / \mathrm{m}^{2}$} \\
\hline baseline & $802 \pm 214$ & $935 \pm 271$ & 0.26 & $814 \pm 205$ & $829 \pm 160$ & 0.83 \\
\hline after & $935 \pm 254$ & $940 \pm 277$ & $886 \pm 219$ & $841 \pm 162$ & & \\
\hline change & $133 \pm 42$ & $5 \pm 63$ & $<0.001$ & $73 \pm 96$ & $12 \pm 39$ & $<0.001$ \\
\hline \multicolumn{7}{|l|}{$\mathrm{PBVI}, \mathrm{mL} / \mathrm{m}^{2}$} \\
\hline baseline & $159 \pm 105$ & $216 \pm 67$ & 0.17 & $213 \pm 57$ & $224 \pm 48$ & 0.68 \\
\hline after & $218 \pm 26$ & $220 \pm 60$ & $215 \pm 38$ & $230 \pm 50$ & & \\
\hline change & $59 \pm 87$ & $4 \pm 63$ & 0.56 & $1 \pm 51$ & $6 \pm 53$ & $<0.001$ \\
\hline Fluid, mL & $600 \pm 0$ & $465 \pm 153$ & $<0.001$ & $460 \pm 195$ & $462 \pm 140$ & 0.84 \\
\hline $\begin{array}{l}\text { Gelatin/HES/ } \\
\text { albumin }\end{array}$ & $1 / 1 / 2$ & $8 / 5 / 10$ & 0.67 & $1 / 3 / 1$ & $5 / 6 / 5$ & 1.0 \\
\hline
\end{tabular}

Mean \pm SD or number of patients where appropriate. Abbreviations: $R$, Responders, NR Non-responders, $C l$ Cardiac index, GEF Global ejection fraction,

$R$ Responding fluid loading step, NR non-responding fluid loading step, CVP Central venous pressure, GEDVI Global end-diastolic volume index, PBVI Pulmonary blood volume index, HES Hydroxyethyl starch, n.a. not applicable. 


\section{Correlations}

Changes in PBVI did not correlate to changes in GEDVI and only the latter related to changes in $\mathrm{CI}$, regardless of GEF ( $r=0.56, \mathrm{P}<0.001$; Figure 1). Changes between 0-90 min in SVI correlated to changes in GEDVI in the low GEF group only $(r=0.70, P=0.03, n=9)$.

\section{Discussion}

Our study suggests that systolic cardiac dysfunction evidenced by a low $\operatorname{GEF}(29,30)$ is common in patients with severe sepsis or septic shock. This dysfunction occurring in $56 \%$ of our patients, independent of cardiac premorbidity, agrees with the literature [1-3,31]. Although this phenomenon might impair fluid responsiveness $(9,18,24)$, our study suggests that fluid responsiveness can be maintained when the heart dilates, even during myocardial depression. In contrast, the optimum GEDVI in patients after cardiovascular surgery ranges from $680-800 \mathrm{~mL} / \mathrm{m}^{2}$ [34-36], and these values may thus not apply in sepsis. A maintained fluid responsiveness at higher GEDVI conforms to the idea that dilatation during sepsis-induced systolic dysfunction is as an aptive response associated with survival by maintaining a relatively high CI [1-3,6,7,9,37]. Indeed, GEDVI was higher prior to responding than to non-responding steps according to $\mathrm{CI} \geq 10 \%$ increases when GEF was low (7 of 9 [77\%] responding steps had a baseline GEDVI $>850 \mathrm{~mL} / \mathrm{m}^{2}$ ). Also, it was not lower in responding than non-responding steps according to $\mathrm{CI} \geq 15 \%$ increases, in contrast to the observations that a low baseline GEDVI, albeit dependent on GEF [25], is more often associated with fluid responsiveness than a relatively high GEDVI $[21,26]$. This confirms that the predictive value for fluid responsiveness of baseline GEDVI or end-diastolic dimensions, rather than changes, is imperfect by its dependency on systolic function, also in sepsis $[21,26,27,38,39]$. That the GEDVI prior to responding fluid loading steps was not lower compared to nonresponding steps when GEF $\geq 20 \%$, can be attributed to a difference in systolic function [26], since CI was lower in the latter. Finally, baseline GEDVI may depend on age and gender [40].

In contrast, we observed that patients with both systolic dysfunction and inability to dilate, were not fluid responsive. The inability to dilate upon systolic dysfunction could comply with the impaired relaxation and diastolic dysfunction found on echocardiography either as an isolated phenomenon or concomitant with systolic dysfunction in $20-60 \%$ of patients with severe sepsis or septic shock [10-16,25]. The phenomenon appeared associated with non-survival and was often transient and reversible in survivors. An additional hypothesis may be the presence of right ventricular dysfunction, in view of the increase in CVP. It cannot be excluded that the presence of predominant right ventricular dysfunction and dilatation limiting left ventricular filling though pericardial constraint may contribute to the lack of fluid responsiveness. Indeed, right ventricular dysfunction caused by moderate pulmonary hypertension (which was not monitored in this study) has been described to limit fluid responsiveness before [6,39]. Out data show that CVP increases upon fluid loading

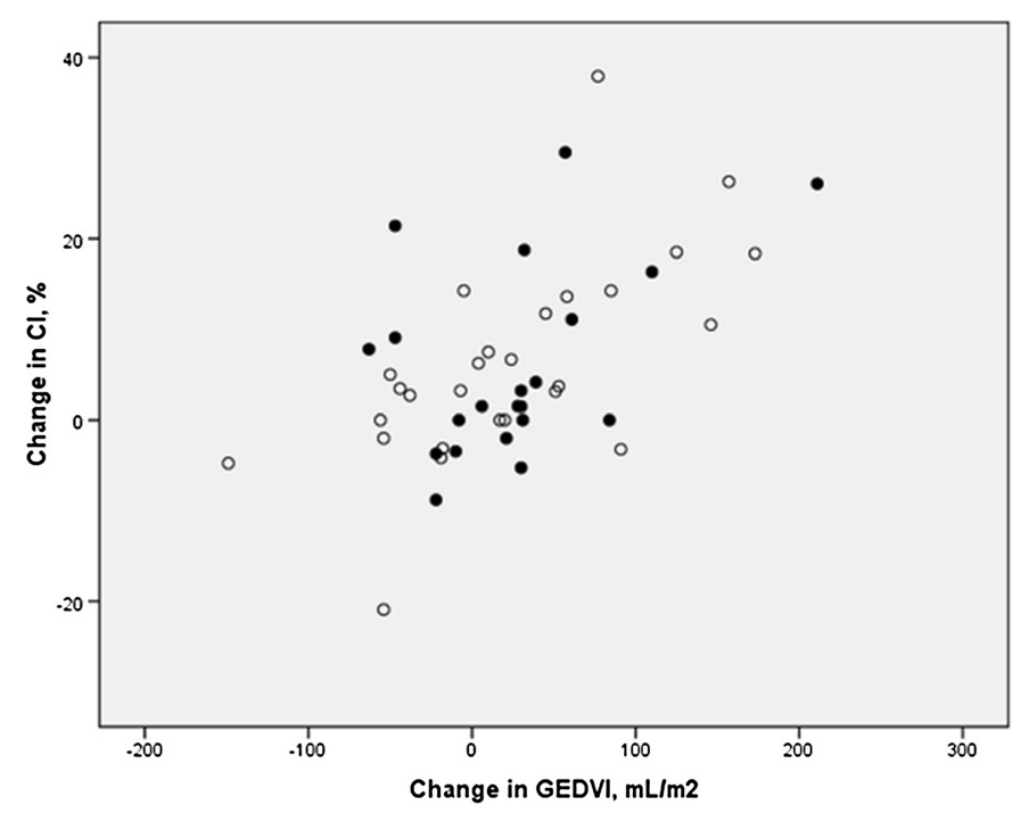

Figure 1 Similar changes in cardiac index $(\mathrm{Cl}, \%)$ versus changes in global end-diastolic volume index $\left(\mathrm{GEDVI}, \mathrm{mL} / \mathrm{m}^{2}\right)$ upon fluid loading steps in patients with low global ejection fraction (open circles, $r=0.65 \mathrm{P}<0.001$ ) and those with near-normal global ejection fraction (closed circles, $r=0.42 \mathrm{P}=0.05$ ) during severe sepsis or septic shock. 
were slightly greater in non-responding than in responding steps which may also point to right ventricular dysfunction and dilatation in some of our patients with low GEF. However, in our study, the increase in CVP was also greater in non-responding than in responding fluid loading steps when GEF was near-normal, which may argue against predominant right ventricular dysfunction in non-responding fluid loading steps of low GEF patients. Since we did not perform operator-dependent, bedside echocardiography simultaneously, to differentiate between right or left ventricular dilatation, we cannot definitively decide on diastolic and/or right ventricular dysfunction in non-responding steps when GEF is low.

Patients with near-normal systolic function were also fluid-responsive by dilatation when operating in the steep part of the cardiac function curve. The dilatation associated with fluid responsiveness, as measured by an increase in GEDVI, is thus independent of systolic cardiac function. Our study partially agrees with data obtained by others suggesting that changes in filling pressures are less helpful in this respect than changes in GEDVI [21-23,26]. Apparently, the phenomenon that impaired systolic function renders filling pressures more important than volumes in the predictive and monitoring value of fluid responsiveness, while the opposite is true when systolic function is relatively normal, after cardiovascular surgery [33], may not apply to sepsis-induced cardiac dysfunction. Otherwise, a higher PEEP level applied in this series than in the previous one [27], may have contributed to the poor predictive value of CVP at low GEF.

Our study has some limitations. The number of patients is relatively small but the study was undertaken to improve interpretation of transpulmonary dilution data with fluid loading in severe sepsis and septic shock rather than to prove benefits thereof. The correlation between changes in GEDVI and CI, regardless of GEF, can be overestimated by mathematical coupling when both are derived from the same thermodilution curve, as argued before [41]. Since both PBVI and GEDVI are also derived, among others, from the same thermodilution curve, mathematical coupling with CI would affect both variables. That PBVI differed from GEDVI in responding to fluid loading and a rise in $\mathrm{CI} \geq 15 \%$ and, in contrast to GEDVI, did not correlate to $\mathrm{CI}$ changes may, however, disfavor mathematical coupling. The CVP changes per step were relatively small in contrast to the overall changes over $90 \mathrm{~min}$. Hence, the right ventricle may have been challenged enough to decide on cardiac responses to fluid loading. In our previous publications we also used hypotension/filling pressure criteria for defining clinical hypovolemia as a common trigger for fluid challenges [24,28,32]; we evaluated the predictive values for fluid responsiveness of other measurements later, to avoid confounding by giving fluids on the basis of allegedly superior predictors of fluid responsiveness than
CVP. We used the delta CVP filling protocol as a safety measure rather than to predict fluid responsiveness. CVP starting values were adjusted for PEEP, in a relatively arbitrary manner, to roughly account for about 50\% transmission of airway pressure, and $\mathrm{CO}$ responses were evaluated at two levels, even though the clinical relevance is unknown.

\section{Conclusions}

In conclusion, our study suggests that in patients with sepsis-induced hypotension and systolic cardiac dysfunction, occurring in about half of patients, fluid responsiveness is maintained by global cardiac dilatation, as measured by transpulmonary dilution-derived GEDVI, rather than by an increase in CVP. Absence of fluid responsiveness in systolic cardiac dysfunction may be explained by diastolic dysfunction and/or concomitant right ventricular dysfunction. Transpulmonary (thermo)dilution-derived GEDVI is more helpful than CVP in monitoring fluid responsiveness and non-responsiveness and their mechanisms in sepsis-induced hypotension, but normal or targeted levels of preload (GEDVI $680-800 \mathrm{~mL} / \mathrm{m}^{2}$ ) may not apply in this condition.

\section{Competing interests}

The authors declare they have no competing interests.

\section{Authors' contributions}

RJT has made substantial contribution to the conception and design of the study including analysis and interpretation of the data as well as drafting the manuscript. ID has been involved in collecting and analysing the data. ABJG conceived of the study, participated in its design and coordination and helped to draft the manuscript and has given final approval of the version to be published. All authors read and approved the final manuscript.

\section{Acknowledgements}

We thank Dr Joanne Verheij for help with collecting data.

\section{Author details}

'Department of Intensive Care, Vrije Universiteit Medical Centre, Amsterdam, The Netherlands. ${ }^{2}$ Department of Intensive Care, Medisch Spectrum Twente, Enschede, The Netherlands. ${ }^{3}$ Department of Intensive Care, Erasmus Medical Centre, Rotterdam, The Netherlands.

Received: 2 November 2012 Accepted: 19 June 2013

Published: 22 June 2013

\section{References}

1. Rabuel C, Mebazaa A: Septic shock: a heart story since the 1960s. Intensive Care Med 2006, 32:799-807.

2. Hunter JD, Doddi M: Sepsis and the heart. Br J Anaesthesia 2010, 104:3-11.

3. Jozwiak M, Persichini R, Monnet X, Teboul JL: Management of myocardial dysfunction in severe sepsis. Semin Respir Crit Care Med 2011, 32:206-214.

4. Bouhemad B, Nicolas-Robin A, Arbelot C, Arthaud M, Féger F, Rouby JJ: Isolated and reversible impairment of ventricular relaxation in patients with septic shock. Crit Care Med 2008, 36:766-774.

5. Parker MM, Shelhamer JH, Bacharach SL, Green MV, Natanson C, Frederick TM, Damske BA, Parrillo JE: Profound but reversible myocardial depression in patients with septic shock. Ann Intern Med 1984, 100:483-490.

6. Schneider AJ, Teule GJ, Groeneveld AB, Nauta J, Heidendal GA, Thijs LG: Biventricular performance during volume loading in patients with early septic shock, with emphasis on the right ventricle: a combined haemodynamic and radionuclide study. Am Heart J 1988, 116:103-112.

7. Parker MM, McCarthy KE, Ognibene FP, Parrillo JE: Right ventricular dysfunction and dilatation similar to left ventricular changes, 
characterize the cardiac depression of septic shock in humans. Chest 1990, 97:126-131.

8. Landesberg G, Gllon D, Meroz Y, Georgieva M, Levin PD, Goodman S, Avidan A, Beeri R, Weisman C, Jaffe AS, Sprung CL: Diastolic dysfunction and mortality in severe sepsis and septic shock. Eur Heart J 2011, 33:895-903.

9. Ognibene FP, Parker MM, Natanson C, Shelhamer JH, Parrillo JE: Depressed left ventricular performance. Response to volume infusion in patients with sepsis and septic shock. Chest 1988, 93:903-910.

10. Poelaert J, Declerck C, Vogelaers D, Colardyn F, Visser CA: Left ventricular systolic and diastolic function in septic shock. Intensive Care Med 1997, 23:553-560.

11. Gödje O, Peyerl M, Seebauer T, Dewald O, Reichart B: Reproducibility of double indicator dilution measurements of intrathoracic blood volume compartments, extravascular lung water, and liver function. Chest 1998, 113:1070-1077.

12. Jardin F, Fourme $T$, Page B, Loubières $Y$, Vieillard-Baron A, Beauchet $A$, Bourdarias JP: Persistent preload defect in severe sepsis despite fluid loading: A longitudinal echocardiographic study in patients with septic shock. Chest 1999, 116:1354-1359.

13. Vieillard Baron A, Schmitt JM, Beauchet A, Augarde R, Prin S, Page B, Jardin F: Early preload adaptation in septic shock? A transesophageal echocardiographic study. Anesthesiology 2001, 94:400-406.

14. Etchecopar-Chevreuil C, François B, Clavel M, Pichon N, Gastinne H, Vignon $P$ : Cardiac morphological and functional changes during early septic shock: a transesophageal echocardiographic study. Intensive Care Med 2008, 34:250-256.

15. Bouhemad B, Nicolas-Robin A, Arbelot C, Arthaud M, Féger F, Rouby J-J: Acute left ventricular dilatation and shock-induced myocardial dysfunction. Crit Care Med 2009, 37:441-447.

16. Sturgess DJ, Marwick TH, Joyce C, Jenkins C, Jones M, Masci P, Stewart D, Venkatesh B: Prediction of hospital outcome in septic shock: a prospective comparison of tissue Doppler and cardiac biomarkers. Crit Care 2010, 14:R44

17. Boyd JH, Forbes J, Nakada T, Walley KR, Russell JA: Fluid resuscitation in septic shock: A positive fluid balance and elevated central venous pressure are associated with increased mortality. Crit Care Med 2011 39:259-265.

18. Weisel RD, Vito L, Dennis RC, Valeri CR, Hechtman HB: Myocardial depression during sepsis. Am J Surg 1977, 133:512-521.

19. Packman Ml, Rackow E: Optimum left heart filling pressure during fluid resuscitation of patients with hypovolemic and septic shock. Crit Care Med 1983, 11:165-169.

20. Dellinger RP, Levy MM, Carlet JM, Bion J, Parker MM, Jaeschke R, Reinhart K, Angus DC, Brun-Buisson C, Beale R, Calandra T, Dhainaut JF, Gerlach H, Harvey M, Marini JJ, Marshall J, Ranieri M, Ramsay G, Sevransky J, Thompson BT, Townsend S, Vender JS, Zimmerman JL, Vincent JL: Surviving Sepsis Campaign: international guidelines for management of severe sepsis and septic shock: 2008. Crit Care Med 2008, 36:296-327.

21. Marx G, Cope T, McCrossan L, Swaraj S, Cowan C, Mostafa SM, Wenstone R, Leuwer M: Assessing fluid responsiveness by stroke volume variation in mechanically ventilated patients with severe sepsis. Eur J Anaesthesiol 2004, 21:132-138.

22. Marik PE, Baram M, Vahid B: Does central venous pressure predict fluid responsiveness? A systematic review of the literature and the tale of seven mares. Chest 2008, 134:172-178.

23. Scheuren K, Wente MN, Hainer C, Scheffler M, Lichtenstern C, Martin E, Schmidt J, Bopp C, Weigand MA: Left ventricular end-diastolic area is a measure of cardiac preload in patients with early septic shock. Eur J Anaesthesiol 2009, 26:759-765

24. Trof RJ, Sukul SP, Twisk JW, Girbes AR, Groeneveld AB: Greater cardiac response of colloid than saline fluid loading in septic and non-septic critically ill patients with clinical hypovolaemia. Intensive Care Med 2010, 36:697-701

25. Hofer CK, Furrer L, Matter-Ensner S, Malpogne M, Klaghofer R, Genoni M, Zollinger A: Volumetric preload measurement by thermodilution: a comparison with transoesophageal echocardiography. $\mathrm{Br} J$ Anaesthesia 2005, 94:748-755.

26. Michard F, Alaya S, Zarka V, Bahloul M, Richard C, Teboul JL: Global end-diastolic volume as an indicator of cardiac preload in patients with septic shock. Chest 2003, 124:1900-1908.

27. Malbrain ML, De Potter TJ, Dits H, Reuter DA: Global and right ventricular end-diastolic volumes correlate better with preload after correction for ejection fraction. Acta Anaesthesiol Scand 2010, 54:622-631.
28. Van der Heijden M, Verheij J, Van Nieuw Amerongen GP, Groeneveld ABJ: Crystalloid or colloid fluid loading and pulmonary permeability, edema and injury in septic and non-septic critically ill patients with hypovolemia. Crit Care Med 2009, 37:1275-1281.

29. Combes A, Berneau J-B, Luyt C-E, Trouillet J-L: Estimation of left ventricular systolic function by single transpulmonary thermodilution. Intensive Care Med 2004, 30:1377-1383

30. Jabot J, Monnet X, Bouchra L, Chemla D, Richard C, Teboul J-L: Cardiac function index provided by transpulmonary thermodilution behaves as an indicator of left ventricular systolic function. Crit Care Med 2009, 37:2913-2918.

31. Ritter S, Rudiger A, Maggiorini M: Transpulmonary thermodilution-derived cardiac function index identifies cardiac dysfunction in acute heart failure and septic patients: an observational study. Crit Care 2009, 13:R133.

32. Trof RJ, Danad I, Reilingh MW, Breukers RM, Groeneveld AJ: Cardiac filling volumes versus pressures for predicting fluid responsiveness after cardiovascular surgery: the role of systolic cardiac function. Crit Care 2011, 15:R73.

33. Levy MM, Fink MP, Marshall JC, Abraham E, Angus D, Cook D, Cohen J, Opal SM, Vincent JL, Ramsay G: SCCM/ESICM/ACCP/ATS/SIS 2001: SCCM/ESICM/ ACCP/ATS/SIS International Sepsis Definitions Conference. Crit Care Med 2003, 31:1250-1256

34. Goepfert MS, Reuter DA, Akyol D, Lamm P, Kilger E, Goetz AE: Goal-directed fluid management reduces vasopressor and catecholamine use in cardiac surgery patients. Intensive Care Med 2007, 33:96-103.

35. Carl M, Alms A, Braun J, Dongas A, Erb J, Goetz A, Goepfert M, Gogarten W, Grosse J, Heller AR, Heringlake M, Kastrup M, Kroener A, Loer SA, Marggraf G, Markewitz A, Reuter D, Schmitt DV, Schirmer U, Wiesenack C, Zwissler B, Spies C: S3 guidelines for intensive care in cardiac surgery patients: hemodynamic monitoring and cardiocirculatory system. Ger Med Sci 2010, 8:1-25.

36. Kirov MY, Kuzkov W, Molnar Z: Perioperative haemodynamic therapy. Curr Opin Crit Care 2010, 16:384-392.

37. Eichhorn V, Goepfert MS, Eulenburg C, Malbrain ML, Reuter DA: Comparison of values in critically ill patients for global end-diastolic volume and extravascular lung water measured by transcardiopulmonary thermodilution: A metaanalysis of the literature. Med Intensiva 2012, 36:467-474.

38. Feissel M, Michard F, Mangin I, Ruyer O, Faller JP, Teboul JL: Respiratory changes in aortic blood velocity as an indicator of fluid responsiveness in ventilated patients with septic shock. Chest 2001, 119:867-877.

39. Wyler von Ballmoos M, Takala J, Roeck M, Porta F, Tueller D, Ganter CC Schröder R, Bracht H, Baenziger B, Jakob SM: Pulse-pressure variation and hemodynamic response in patients with elevated pulmonary artery pressure: a clinical study. Crit Care 2010, 14:R111.

40. Wolf S, Riess A, Landscheidt JF, Lumenta CB, Friederich P, Schürer L: Global end-diastolic volume acquired by transpulmonary thermodilution depends on age and gender in awake and spontaneously breathing patients. Crit Care 2009, 13:R202.

41. Breukers RM, de Wilde RB, van den Berg PC, Jansen JR, Faes TJ, Twisk JW, Groeneveld $A B$ : Assessing fluid responses after coronary surgery: role of mathematical coupling of global end-diastolic volume to cardiac output measured by transpulmonary thermodilution. Eur J Anaesthesio/ 2009, 26:954-960

doi:10.1186/1471-2253-13-12

Cite this article as: Trof et al:: Global end-diastolic volume increases to maintain fluid responsiveness in sepsis-induced systolic dysfunction. BMC Anesthesiology 2013 13:12. 